\title{
Agenesis of Dorsal Pancreas: Incidental Diagnosis Made with Contrast Enhanced Imaging Done for Other Pathologies
}

\author{
Dwivedi $\mathrm{AN}^{1}$ and Tripathi $\mathrm{K}^{2}$ \\ ${ }^{1}$ Department of Radiodiagnosis and Imaging, Institute of Medical Sciences, Banaras Hindu \\ University, India \\ ${ }^{2}$ Department of Medicine, Institute of Medical Sciences, Banaras Hindu University, India
}

*Corresponding author: Amit Nandan Dhar Dwivedi (M.D), Department of Radiodiagnosis and Imaging, Institute of Medical Sciences, Banaras Hindu University, India, Tel: +919793856930; Email: amitnandan21@yahoo.com

\section{Case Report \\ Volume 4 Issue 3}

Received Date: August 15, 2020

Published Date: September 15, 2020

DOI: $10.23880 /$ crij-16000179

\section{Abstract}

Dorsal pancreatic agenesis is an extremely rare congenital anomaly of pancreas. It results from an embryological failure of the dorsal pancreatic bud to form the body and tail of the pancreas. Nowadays use of contrast enhanced computerized scan for various pathologies have made the diagnosis more frequent as compared to the past. This rare anomaly is more frequently diagnosed due to improved scanning protocol and sophisticated software. We report the contrast enhanced imaging details of this rare condition which was incidentally diagnosed while scanning done for unrelated pathology.

Keywords: Dorsal Pancreas; Congenital anomaly; Abdomen

\section{Introduction}

Agenesis of dorsal pancreas is an extremely rare congenital anomaly of pancreas. It may be partial or complete agenesis dorsal pancreas. A few case reports have been published in the literature. We are reporting the CT appearance of this condition in two cases. The patients with dorsal pancreatic agenesis can be asymptomatic or present with abdominal pain; weight loss; diabetes mellitus; pancreatitis; bile duct obstruction; duodenal obstruction; or rarely pancreatic exocrine insufficiency and pancreatic adenocarcinoma.

\section{Case Report}

Diagnosis of agenesis of dorsal pancreas was made in two patients over a period of one year (February to January). The first case was thirty year old female presented with pain in lower abdomen. On ultrasonography examination, the head of pancreas was visible. The pancreatic body and tail were not identified and space anterior to splenic vein was filled with ill-defined echogenic structures. Horseshoe kidney was also noted in this patient. On CECT, the absence of body and tail of pancreas was demonstrated. The space anterior to splenic vein was occupied by jejunal loops (dependent intestine sign). Head and uncinate process of pancreas were normal in size, density and enhancement pattern (Figure 1).

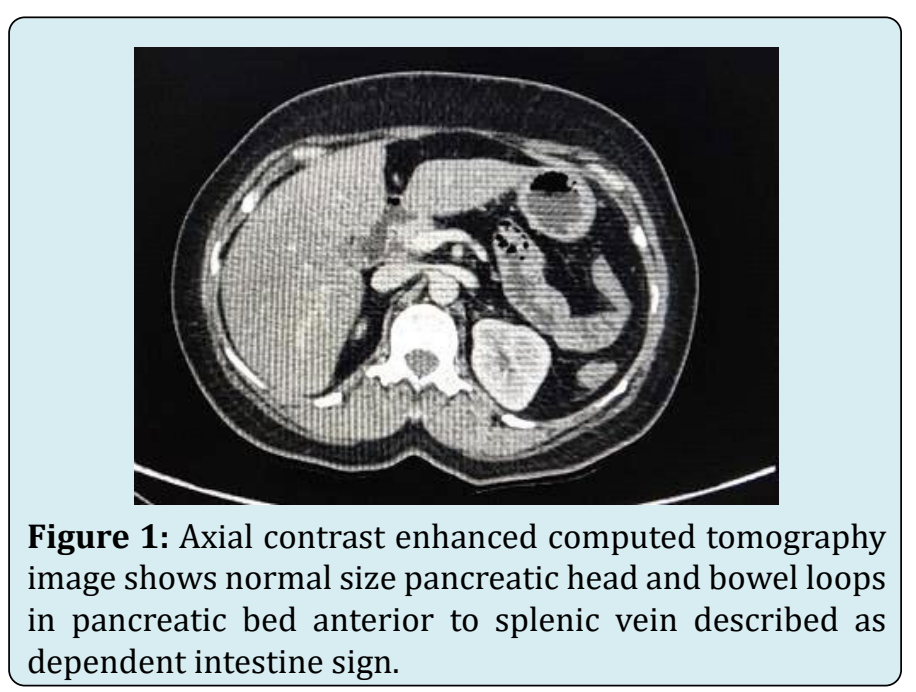




\section{Clinical Radiology \& Imaging Journal}

Second case was fifty year old female, known diabetic since three years and scanning done to evaluate for possible diagnosis of carcinoma caecum on CECT. In this patient, the absence of body and tail of pancreas was detected incidentally on CECT. The space anterior to splenic vein was occupied by stomach (dependent stomach). Head and uncinate process of pancreas were normal in size, density and enhancement pattern (Figure 2).

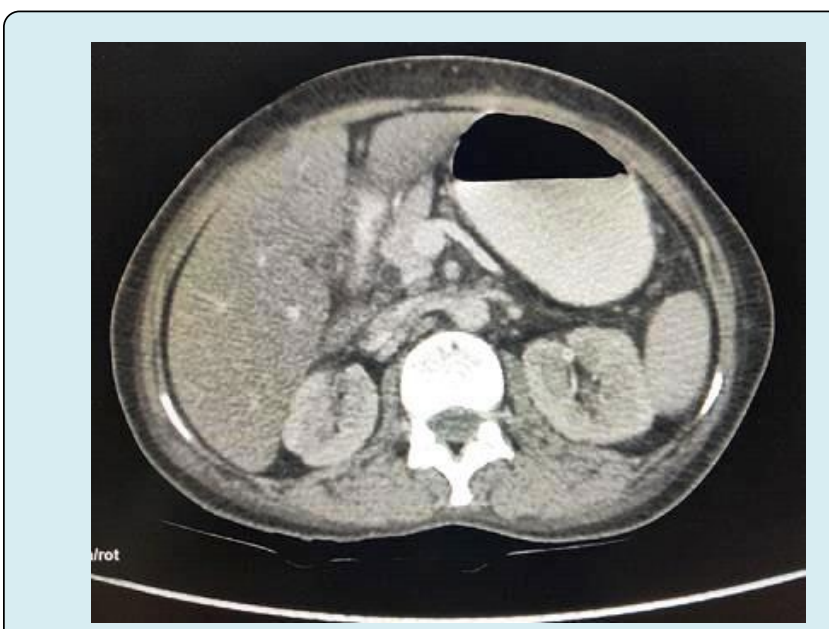

Figure 2: Axial contrast enhanced computed tomography image shows normal size pancreatic head and pancreatic bed anterior to splenic vein is filled by stomach described as dependent stomach sign.

\section{Discussion}

The pancreas appears at approximately 5th week of gestation in the form of two separate endodermal buds (ventral and dorsal) from caudal region of the embryonic foregut. The fusion of ventral and dorsal bud occurs at around 6-7week of gestation. The ventral bud gives rise to lower portion of the head and the uncinate process of pancreas. The dorsal bud gives rise to upper head; neck; body; and tail of pancreas. The ventral bud drains through duct of Santorini and the dorsal bud drain through duct of Wrisung. These two ducts join to form major pancreatic duct which in turn joins the common bile duct and drains into ampulla of Vater [1]. Complete agenesis of pancreas and agenesis of ventral pancreas are incompatible with life [2]. Agenesis of dorsal pancreas is an extremely rare congenital anomaly. In complete agenesis, the neck, body, and tail of pancreas, duct of Santorini, and minor duodenal papilla are absent. In partial agenesis of dorsal pancreas, there is a remnant of duct of Santorini and minor duodenal papilla [3].

The cause of agenesis of dorsal pancreas is unknown. Primary dysgenesis or ischemic events in the developing pancreas is the possible explanations [4]. It is usually sporadic.
It can occur as an autosomal dominant or $\mathrm{x}$-linked dominant inheritance or with polysplenia/ heterotaxy syndrome [5]. Agenesis of dorsal pancreas can be associated with annular pancreas or with congenital defects like congenital defects like congenital hydrocephalus, hepato-mesenteric trunk, horseshoe kidney, and retro-aortic left renal vein [6]. Many patients with agenesis of dorsal pancreas are asymptomatic. In some patients it may be symptomatic. The symptomatic (92.9\%) patients will present with abdominal pain [7]. The pain may be due to pancreatitis; duodenal obstruction; sphincter of Oddi dysfunction or autonomic neuropathy. The Sphincter of Oddi dysfunction or dyskinesia, compensatory hypertrophy and hypersecretion of remaining ventral pancreas with raised intraductal pressure have been proposed to explain pancreatitis [8-10]. Another common clinical manifestation association with agenesis of dorsal pancreas is diabetes mellitus. Development of diabetes mellitus may be due to the absence of the islet cells which usually reside in the body and tail of the pancreas [11].

In agenesis of dorsal pancreas, the body and tail are absent and pancreatic bed anterior to splenic vein is occupied by stomach and bowel loops - dependent stomach and dependent intestine sign on CT [12]. On ultrasonography examination the stomach and intestine loops when air filled appear as an echogenic structure in the pancreatic bed this may interfere with adequate visualization of the body and tail of pancreas. This may be misinterpreted as normal pancreas on ultrasonography examination. When ultrasonography examination is suboptimal, before going for CT and MRI ultrasonography examination can be performed after adequately distending the stomach with water to get good window for proper visualization of the pancreatic body and tail. If this method fails CT and MRI studies are useful for evaluating pancreatic body and tail. The dependent stomach and dependent intestine sign were observed in our cases. CT equivalent of dependent stomach and dependent intestine sign can also be observed on MRI. T2 weighted sequence is better than T1 weighted fat saturated sequence for depicting bowel loops In pancreatic bed as collapsed small bowel loops in the pancreatic bed appear isointense with the pancreatic head and may be misinterpreted as normal pancreatic body and tail [13]. The pseudo agenesis (atrophy of body and tail of the pancreas secondary to chronic pancreatitis) and distal pancreatic lipomatosis can be considered as differential diagnosis of agenesis of dorsal pancreas [14,15]. Dorsal duct is absent or if present it will be very short in dorsal agenesis and it is usually present in lipomatosis and pseudo agenesis. Demonstration of dorsal duct and dependent stomach and dependent intestine sign on multidetector CT scan allow differentiation of agenesis of dorsal pancreas from distal lipomatosis [13]. ERCP is considered to be the gold standard investigation for detailed evaluation of the pancreatic tree, however it is invasive, operator dependent, requires 


\section{Clinical Radiology \& Imaging Journal}

radiation exposure and risk of iatrogenic pancreatitis. MRCP may be the choice of investigation for confirmation of diagnosis of agenesis of dorsal pancreas, as it is non-invasive and accurately depicts the pancreatic duct morphology and parenchyma in the same examination [16].

\section{Conclusion}

Agenesis of dorsal pancreas is a rare anomaly. However, with the availability of cross-sectional imaging, a greater number of cases have been detected and reported in the last decade. CT is the readily available as well as better diagnostic tool in diagnosis of agenesis of dorsal pancreas.

\section{References}

1. Pandol SJ (2010) The Exocrine Pancreas. San Rafael (CA): Morgan \& Claypool Life Sciences. Pancreatic Embryology and Development.

2. Voldsgaard P, Kryger-Baggesen N, Lisse I (1994) Agenesis of pancreas. Acta Paediatr 83(7): 791-793.

3. Fukuoka K, Ajiki T, Yamamoto M, Fujiwara H, Onoyama H, et al. (1999) Complete agenesis of the dorsal pancreas. J Hepatobiliary Pancreat Surg 6: 94-97.

4. Macari M, Giovanniello G, Blair L, Krinsky G (1998) Diagnosis of agenesis of the dorsal pancreas with MR pancreatography. AJR Am J Roentgenol 170(1): 144-146.

5. Sener RN, Alper H (1994) Polysplenia syndrome: A case associated with transhepatic portal vein, short pancreas, and left inferior vena cava with hemiazygous continuation. Abdom Imaging 19: 64-66.

6. Vijayaraghavan SB, Gouru S, Senthi S (2013) Sonographic features of agenesis of dorsal pancreas. Indian J Radiol Imaging 23(2): 179-182.

7. Sempere L, Aparicio JR, Martinez J, Casellas JA, Dee
Madaria E, et al. (2006) Role of endoscopic ultrasound in diagnosis of agenesis of the dorsal pancreas. J Pancreas 7: 411-416.

8. Klein WA, Dabezies MA, Friedman AC, Caroline DF, Boden GH, et al. (1994) Agenesis of dorsal pancreas in a patient with weight loss and diabetes mellitus. Dig Dis Sci 39: 1708-1713.

9. Nishimori I, Okazaki K, Morita M, Miyao M, Sakamoto $Y$, et al. (1990) Congenital hypoplasia of the dorsal pancreas: With special reference to duodenal papillary dysfunction. Am J Gastroenterol 85(8): 1029-1033.

10. Lechner GW, Read RC (1996) Agenesis of the dorsal pancreas in anadult diabetic presenting with duodenal ileus. Ann Surg 163(2): 311-314.

11. Pasaoglu L, Vural M, Hatipoglu HG, Tereklioglu G, Koparal S (2008) Agenesis of the dorsal pancreas. World J Gastroenterol 14(18): 2915-2916.

12. Mohapatra M, Mishra S, Dalai PC, Acharya SD, Nahak B, et al. (2012) Imaging findings in agenesis of the dorsal pancreas. Report of three cases. JOP 13(1): 108-114.

13. Mohapatra M, Mishra S, Dalai PC, Acharya SD, Nahak $B$, et al. (2012) Imaging findings in agenesis of dorsal pancreas. Report of three cases. JOP 13(1): 108-114.

14. Karcaaltincaba M (2006) CT differentiation of distal pancreas fatreplacement and distal pancreas agenesis. Surg Radiol Anat 28: 637-641.

15. Uygur-Bayramicli O, Dabak R, Kilicoglu G, Dolapcioglu C, Oztas D (2007) Dorsal pancreatic agenesis. JOP 8: 450452.

16. Balakrishnan V, Narayanan VA, Siyad I, Radhakrishnan L, Nair P (2006) Agenesis of the dorsal pancreas with chronic calcific pancreatitis. Case report, review of the literature and genetic basis. JOP 7: 651-659. 